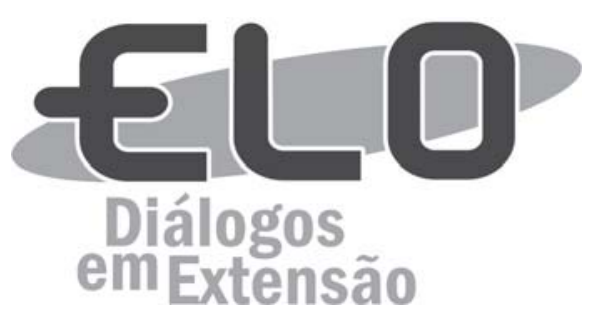

\title{
Educação alimentar e nutricional para o estímulo do consumo de pescados por escolares: relato de experiência
}

\author{
Viviane Ferreira dos Santos ${ }^{1}$, Izabel Alice de Araújo ${ }^{1}$, Caroline Roberta Freitas Pires ${ }^{2}$, \\ Hellen Christina de Almeida Kato ${ }^{3}$, Diego Neves de Sousa ${ }^{4}$
}

\begin{abstract}
Resumo: A escola é indiscutivelmente o melhor agente para promover a educação alimentar, uma vez que é na infância e na adolescência que se fixam atitudes e práticas alimentares difíceis de modificar na idade adulta. Diante disso, esse trabalho objetivou utilizar atividades lúdicas para empoderar os escolares quanto à aquisição, composição e consumo de peixe. Trata-se de um relato de experiência de caráter descritivo que baseia-se no desenvolvimento de um programa de atividades de Educação Alimentar e Nutricional (EAN) com 31 escolares do $5^{\circ}$ ano matriculados em uma escola da rede municipal do município de Palmas. As atividades de EAN foram desenvolvidas em 3 encontros com duração de 40 minutos. Nos encontros foram utilizadas diferentes metodologias, como exposição dialogada, dinâmica de grupo, colagens em cartazes e oficina culinária. A avaliação do conhecimento deu-se de forma objetiva com a aplicação de um pré e pós-teste. As atividades lúdicas oportunizaram a construção de conhecimento sobre aquisição, composição e consumo dos peixes, demonstrando a aplicabilidade das oficinas.
\end{abstract}

Palavras-chave: Intervenção Nutricional. Alimentação Infantil. Pescado.

Área Temática: Teorias e Metodologias em Extensão. Políticas Públicas.

\section{Food and nutrition education for the stimulation of consumption of fish in schoolchildren: experience report}

\begin{abstract}
The school is undoubtedly the best agent for promoting food education, since it is in childhood and adolescence that food habits and practices that are difficult to change in adulthood are set. In view of this, this work aimed to use play activities to empower the students regarding the acquisition, composition and consumption of fish. This is a descriptive experience report that is based on the development of a program of EAN activities with 315 th grade students enrolled in a municipal school in the municipality of Palmas. EAN activities were developed in 3 meetings lasting 40 minutes. In the meetings were used different methodologies, such as dialogues, group dynamics, posters and culinary workshop collages. Knowledge evaluation was done objectively with the application of a pre-and post-test. The ludic activities allowed the construction of knowledge on the acquisition, composition and consumption of the fish, demonstrating the applicability of the workshops.
\end{abstract}

Keywords: PNutritional Intervention. Infant Feeding. Fish.

\section{Educación alimentaria y nutricional para el estimulo del consumo de pescados por escolares: relato de experiência}

Resumen: La escuela es indiscutiblemente el mejor agente para promover la educación alimentaria, ya que es en la infancia y en la adolescencia que se fijan actitudes y prácticas alimentarias difíciles de modificar en la edad adulta. Frente a ello, este trabajo tuvo como objetivo utilizar actividades lúdicas para empoderar

\footnotetext{
${ }^{1}$ Graduada em Nutrição pela Universidade Federal do Tocantins - UFT, Palmas - TO, Brasil (vivianefsnutri@gmail.com)

${ }^{2}$ Professora Doutora do curso de Nutrição da Universidade Federal do Tocantins - UFT, Palmas -TO, Brasil.

${ }^{3}$ Mestre em Ciência e Tecnologia de Alimentos da Universidade Federal do Pará

${ }^{4}$ Doutorando em Desenvolvimento Rural pela Universidade Federal do Rio Grande do Sul (UFRGS)
} 
a los escolares en cuanto a la adquisición, composición y consumo de pescado. Se trata de un relato de experiencia de carácter descriptivo que se basa en el desarrollo de un programa de actividades de EAN con 31 escolares del $5^{\circ}$ año matriculados en una escuela de la red municipal del municipio de Palmas. Las actividades de EAN se desarrollaron en 3 encuentros con una duración de 40 minutos. En los encuentros se utilizaron diferentes metodologías, como exposición dialogada, dinámica de grupo, collages en carteles y taller culinario. La evaluación del conocimiento se dio de forma objetiva con la aplicación de un pre y post-test. Las actividades lúdicas oportunizaron la construcción de conocimiento sobre adquisición, composición y consumo de los peces, demostrando la aplicabilidad de los talleres.

Palabras clave: Intervención Nutricional. Alimentación infantil. Fish.

\section{Introdução}

O Programa Nacional de Alimentação Escolar (PNAE), criado na década de 50, é o mais antigo programa social do Governo Federal na área de alimentação e nutrição (BRASIL, 2013). Gerenciado pelo Fundo Nacional de Desenvolvimento da Educação (FNDE) o programa dispõe de recursos e mecanismos que contribuem para a oferta de uma alimentação adequada e balanceada aos estudantes da rede pública durante o período em que permanecerem no ambiente escolar (SILVA, 2017).

Além disso, o programa visa ofertar refeições que compreendam o uso de alimentos variados, seguros, que respeitam a cultura, as tradições e os hábitos alimentares saudáveis (BRASIL, 2013).

A alimentação escolar contribui para a aprendizagem e o melhor rendimento dos estudantes garantindo um adequado crescimento e desenvolvimento físico, além do aumento do rendimento intelectual (SILVA, 2017).

Nessa perspectiva, o PNAE pode se constituir em um instrumento pedagógico para o desenvolvimento de ações de segurança alimentar e nutricional, tendo nas escolas um espaço estratégico para garantir o fortalecimento das práticas de educação alimentar e nutricional (CASEMIRO et al., 2014).

A escola é um espaço estratégico para a promoção da saúde, nela encontram-se os meios necessários para o desenvolvimento de atividades de educação alimentar e nutricional, ferramenta indispensável para a fixação de hábitos saudáveis na infância que se consolidaram na vida adulta (TURANO, 1990).

Nesse sentido Rossi et al. (2008) afirma que experiências precoces e interação contínua com o alimento influenciam nas preferências alimentares, hábitos e atitudes exibidas na vida adulta, pois quanto mais precoce for adotado um estilo de vida saudável, menor será o risco de doenças crônicas não transmissíveis no futuro.

Heberle (2011) enfatiza a adoção de atividades lúdicas como um importante instrumento de auxílio na educação alimentar e nutricional (EAN), sejam eles jogos, quebra-cabeça, entre outros elementos que além de promover conhecimento científico, ainda proporcionam prazer no envolvimento dos alunos com a aula, interferindo de maneira sutil no estilo de vida e hábitos alimentares.

A preferência alimentar das crianças na idade pré-escolar conduz ao consumo de alimentos com quantidade elevada de carboidrato, açúcar, gordura e sal, e baixo consumo de alimentos de maior qualidade nutricional, como; peixes, vegetais e frutas (KREBS-SMITH et al., 1996).

O peixe é um dos alimentos mais indicados para a alimentação escolar, pois tem excelentes propriedades nutricionais, sendo uma das principais fontes de proteína de origem animal pelo elevado valor biológico decorrente da alta sensibilidade à digestão e a composição balanceada de aminoácidos (ORDÓNEZ, 2005).

Além disso, apresenta vitaminas (A e D) e minerais tais como o cálcio, ferro e fósforo, sendo também fonte de ácidos graxos essenciais, como o ômega-3, que estão associados à redução do risco de doenças cardiovasculares e a funções importantes nas fases iniciais do desenvolvimento humano (BRAGANÇA, 2016).

Mesmo o Brasil sendo um importante produtor de pescado, o seu consumo é relativamente inferior a média mundial, de $16 \mathrm{~kg}$ por habitante, enquanto o brasileiro por ano consume apenas $9 \mathrm{~kg}$, índice considerado baixo pela Organização Mundial da Saúde, que recomenda pelo menos $12 \mathrm{~kg}$ por habitante (SARTORI e AMANCIO, 2012).

Nessa perspectiva o Ministério da Educação e o da Agricultura se une para inserir o pescado nas escolas, visando aumentar a renda das comunidades pesqueiras e aquícolas, assim como melhorar os valores nutricionais da alimentação escolar nas regiões produtoras de pescado (NETO, 2015). 
Nesse sentido, o presente estudo foi desenvolvido com o objetivo de incentivar e despertar nos escolares o interesse pelo consumo do pescado, abordando seus principais nutrientes e benefícios, utilizando propostas de atividades lúdicas e ilustrativas para aquisição de hábitos e práticas alimentares saudáveis.

\section{Metodologia}

Trata-se de um relato de experiência de caráter descritivo, obtido com base em ações de Educação Alimentar e Nutricional como parte do projeto desenvolvido a partir de um trabalho maior intitulado "Transferência de tecnologia para inserção do pescado da agricultura familiar na alimentação escolar", realizado pela Empresa Brasileira de Pesquisa Agropecuária (EMBRAPA), pelo Grupo de Estudos em Educação para Promoção da Saúde (GEPEPS) do Centro Universitário Luterano de Palmas (CEULP) e pelo curso de Nutrição da Universidade Federal do Tocantins (UFT), cujo principal objetivo consiste na busca de estratégias para inserção do pescado na alimentação escolar, estimulando hábitos saudáveis para a promoção da saúde.

As atividades de Educação Alimentar e Nutricional foram realizadas com 31 alunos do $5^{\circ}$ ano matriculados no segundo semestre do ano letivo de 2017 da escola Municipal Monteiro Lobato do município de Palmas, no estado do Tocantins.

Participaram das atividades de EAN todas as crianças devidamente autorizadas por pais ou responsáveis mediante a assinatura do Termo de Consentimento Livre e Esclarecido - TCLE.

\section{Etapa 1:}

Para a concretização das atividades, inicialmente foi realizada uma reunião com a diretora da escola e com as professoras da turma selecionada. Na reunião foi apresentado o objetivo do projeto e as ações que seriam desenvolvidas com os alunos.

\section{Etapa 2:}

Na segunda etapa foi realizada uma atividade prática para que os alunos pudessem conhecer as características de um peixe. As crianças inicialmente realizaram o pré-teste que tinha como objetivo principal identificar o conhecimento prévio dos escolares sobre as principais qualidades que um peixe fresco deve apresentar no ato da compra. Essa ação buscou levar aos alunos conhecimento acerca da aquisição do peixe, tornando-os ativos no momento da compra, promovendo a adoção de hábitos alimentares saudáveis na fase escolar.

Para a condução dessa atividade foram adquiridos dois peixes, sendo um fresco e o outro parcialmente deteriorado com características indesejáveis de consumo. Os mesmos foram colocados em uma bandeja de polietileno branca recoberta com gelo para o apoio e manutenção do peixe.

Em seguida foram analisados os seguintes atributos de qualidade: olhos, brânquias, escamas, curvatura, odor e musculatura.

A turma foi dividida em três grupos para que os alunos pudessem participar mais ativamente da atividade "olhando e sentindo". Foi realizada uma exposição dialogada com os alunos que se encontravam dispostos ao redor de uma mesa centralizada na sala, permitindo que eles pudessem participar da discussão visualizando todos os aspectos abordados durante a ação desenvolvida.

Após a abordagem de todos os aspectos que devem ser analisados no ato da compra do pescado, cada criança recebeu um par de luvas para que pudessem apalpá-los, aumentando a sua autonomia na escolha. Finalizada a prática, foram distribuídas figuras ilustrativas de peixes, para que os mesmos fixassem as modificações dos atributos que conferem qualidade e frescor a esse alimento.

Encerrando o momento, os alunos receberam as fichas contendo o pós-teste com o objetivo de avaliar o conhecimento adquirido com a atividade.

\section{Etapa 3:}

A terceira etapa consistiu na aplicação de um pré-teste que continha perguntas referentes à composição química do peixe. O pré-teste foi conduzido a partir de uma explanação dialógica, reforçando os conhecimentos dos discentes em relação aos nutrientes presentes nos peixes e o benefício 
de sua ingestão para a saúde. Nessa etapa os alunos inicialmente receberam um cartão e o comando para que pudessem desenhar um peixe. Nesse momento foi despertada a criatividade de cada criança. Com o desenho pronto cada aluno recebeu um balão para colar o seu "peixe". No interior do balão cheio continha um pedaço de papel com a escrita de alguns nutrientes da sua composição (proteína, ômega 3, ferro, cálcio, fósforo, Vitamina D e A).

Em seguida, foi dado o comando para que cada um estourasse o seu balão e encontrasse o nutriente escrito no papel. Para a conclusão da atividade, foi montado um painel com os desenhos confeccionados pelos alunos e os nutrientes citados.

Finalizada essa etapa, foi feita a explanação sobre a função e importância de cada nutriente presente na carne do peixe. Além disso, também foram apontadas outras fontes dos nutrientes além do pescado, ampliando a visão dos alunos acerca das fontes de nutrientes e adoção de práticas alimentares saudáveis.

Após a exposição os alunos receberam o pós-teste para a conclusão dessa etapa.

\section{Etapa 4:}

Finalizada a abordagem sobre a escolha do peixe e da sua composição, a etapa final foi denominada “vamos comer o peixe?". Nessa etapa os alunos participaram ativamente da condução de uma receita que tinha em sua composição o peixe. Foi elaborada uma receita de Torta de Atum. Cada criança contribuiu no desenvolvimento da receita, adicionando os ingredientes e fazendo a mistura para obtenção do produto final. Nesse momento, ressaltou-se a importância das crianças se sentirem responsáveis pelo preparo do alimento, incentivando o consumo do mesmo. Terminada a preparação da torta os alunos fizeram a degustação.

\section{Resultados e discussão}

Das ações educativas participaram 31 escolares do $5^{\circ}$ ano do ensino fundamental, com faixa etária de 9 a 11 anos, sendo composta de 13 (42\%) do sexo feminino e 18 (58\%) do sexo masculino.

No primeiro momento, foi perguntado aos alunos se eles sabiam como escolher o peixe na hora da compra, para isso foi elaborado um pré-teste, com pontos importantes a serem observados na sua aquisição (Tabela 01).

Segundo Kato (2014) devem ser observados os seguintes aspectos: a pele deve estar brilhante, as escamas íntegras e bem aderidas à pele, os olhos transparentes, brilhantes com cores vivas e ligeiramente salientes, as brânquias avermelhadas e úmidas, o abdômen roliço, firme e elástico e o odor moderado, característico de cada espécie.

As figuras 1 e 2 mostram as características dos dois peixes utilizados e a participação do alunos na oficina intitulada como "olhando e sentido".
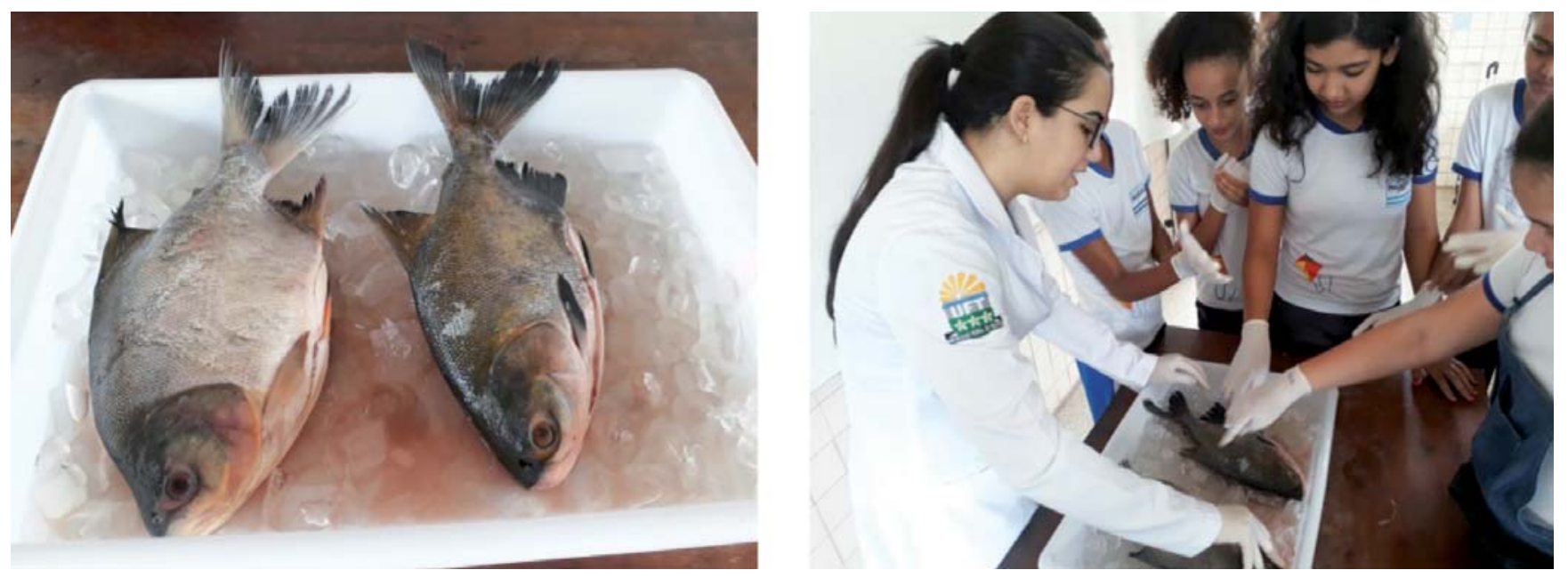

Figura 1 - Peixes em diferentes estados de conservação. Figura 2 - Alunos participando da oficina olhando e sentindo.

Fonte: arquivo próprio. 
Ao avaliar os resultados obtidos após a aplicação do pós-teste observou-se um aumento no número de acertos das questões a cerca dos atributos avaliados (Tabela 1).

A utilização de pré e pós-teste, permite avaliar a compreensão e assimilação do conteúdo abordado durante as ações. Na tabela 01 é possível observar uma melhora considerável de acertos no pós-teste para todas as perguntas do questionário, mostrando uma compensação positiva e fixação do conteúdo por parte dos alunos, fato este comprovado ao avaliar os atributos olhos, brânquias, curvatura e cheiro (100\% de acertos).

Tabela 1 - Avaliação do conhecimento antes e após atividade educativa sobre os atributos que interferem na escolha do peixe.

\begin{tabular}{|c|c|c|c|c|}
\hline & \multicolumn{2}{|c|}{ Pré Teste } & \multicolumn{2}{|c|}{ Pós Teste } \\
\hline & Erros & Acertos & Erros & Acertos \\
\hline Pele & $16,13 \%$ & $83,87 \%$ & $3,23 \%$ & $96,77 \%$ \\
\hline Escamas & $6,45 \%$ & $93,55 \%$ & $3,23 \%$ & $96,77 \%$ \\
\hline Olhos & $25,81 \%$ & $74,19 \%$ & $0 \%$ & $100 \%$ \\
\hline Brânquias & $38,71 \%$ & $61,29 \%$ & $0 \%$ & $100 \%$ \\
\hline Musculatura & $38,71 \%$ & $61,29 \%$ & $9,68 \%$ & $90,32 \%$ \\
\hline Curvatura & $51,61 \%$ & $48,39 \%$ & $0 \%$ & $100 \%$ \\
\hline Cheiro & $16,13 \%$ & $83,87 \%$ & $0 \%$ & $100 \%$ \\
\hline
\end{tabular}

Fonte: elaborada pelos autores (2017).

Da mesma forma, Fonseca et al. (2002), utilizando o mesmo instrumento de avaliação em atividades lúdicas, obtiveram resultados satisfatórios ao avaliar a compreensão e fixação do conteúdo ministrado e, o nível de aproveitamento passou de $16,7 \%$ no pré-teste para $77,8 \%$ no pós-teste.

Resultado semelhante foi obtido por Vivas e Sequeda (2003) ao avaliar um jogo de tabuleiro como mediador da aprendizagem, no qual os conhecimentos e habilidades foram menores no préteste (6,5 e 18,4 pontos) que no pós (8,25 e 22,9 pontos). Aproximadamente $86 \%$ dos estudantes tiveram maior aprendizado e incorporação do conteúdo após a utilização de jogos.

Segundo Winnicott (1975), a adoção de atividades lúdicas irá construir uma bagagem cultural para a criança e se incorporar na fase adulta. Dessa maneira, espera-se que após adquirido o conhecimento sobre a escolha do peixe, a criança possa levá-lo pela sua vida, de forma que seja um conhecimento fixo e não temporário.

Na segunda etapa das atividades os alunos foram estimulados a desenvolverem as suas habilidades artísticas, colorindo e confeccionando desenhos de peixes de acordo com sua imaginação.

De acordo com Garrocho (1990), a arte pode ser utilizada como técnica de ensino para escolares, contribuindo para o desenvolvimento do aprendizado, já que é através da interação da criança com o meio que se inicia a aprendizagem.

Para a realização de atividades de educação alimentar e nutricional existem técnicas como dinâmicas em grupos, exposições orais, músicas, vídeos e dramatizações, havendo a necessidade de planejamento adequado a fim de atender o público e os objetivos propostos (COSTA et al., 2016).

Nessa etapa, a exposição sobre a composição química do peixe foi realizada de forma dialógica na qual os escolares estiveram envolvidos na atividade de educação alimentar e nutricional, tendo a oportunidade de compartilhar suas experiências e manifestar suas opiniões. Paulo Freire (2007) enfatiza que a metodologia dialógica só promove a ampliação da visão do mundo quando há uma relação mediada pelo diálogo.

As figuras 3 e 4 mostram os desenhos de peixes feitos pelos alunos e também o mural elaborado com os nutrientes de sua composição.

Na tabela 2, podemos verificar que os escolares apresentaram pouco conhecimento no pré-teste em relação aos nutrientes e suas respectivas funções antes da atividade educativa.

No entanto, é válido ressaltar que mesmo após a exposição do conteúdo, o percentual de acertos ainda permaneceu baixo, o que justifica a necessidade de realização de outras atividades de educação alimentar e nutricional com esse tema. 

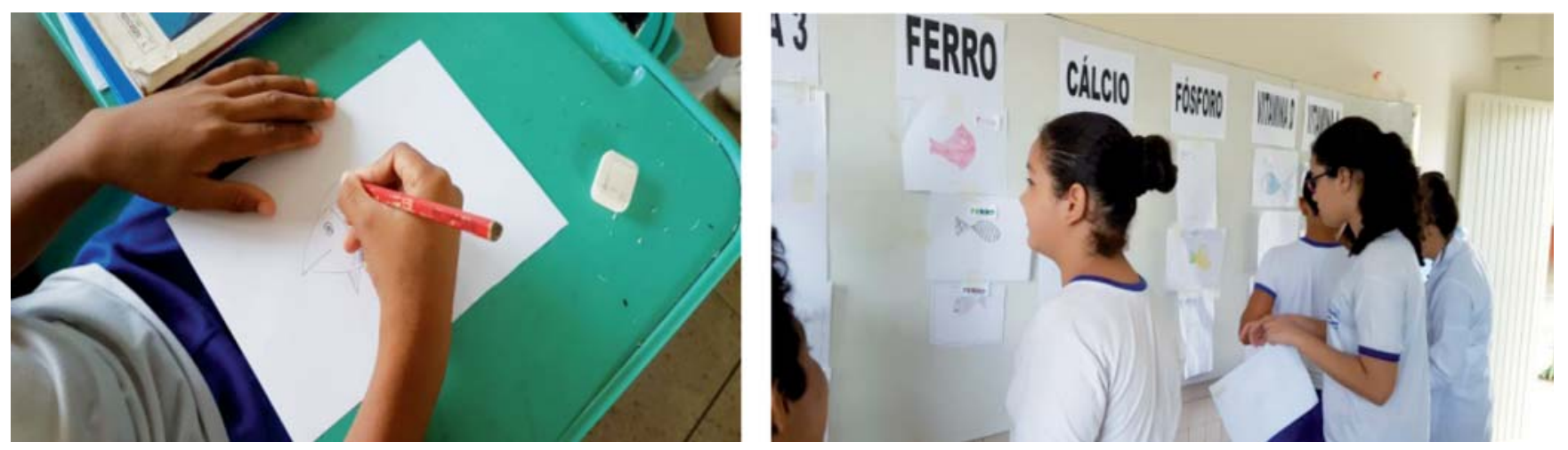

Figura 3 - Arte dos alunos - Desenho do peixe. Figura 4 - Mural com os nutrientes e os desenhos dos peixes. Fonte: arquivo próprio

Tabela 2 - Avaliação do conhecimento antes e após atividade educativa sobre a composição nutricional do peixe.

\begin{tabular}{lccccc}
\hline & \multicolumn{2}{c}{ Pré Teste } & & \multicolumn{2}{c}{ Pós Teste } \\
\cline { 2 - 3 } \cline { 5 - 6 } & Erros & Acertos & & Erros & Acertos \\
\hline Proteína & $87,50 \%$ & $12,50 \%$ & $28,13 \%$ & $71,87 \%$ \\
Ômega 3 & $78,13 \%$ & $21,87 \%$ & & $46,88 \%$ & $53,12 \%$ \\
Ferro & $68,75 \%$ & $31,25 \%$ & & $46,88 \%$ & $53,12 \%$ \\
Cálcio & $84,38 \%$ & $15,62 \%$ & & $50 \%$ & $50 \%$ \\
Fósforo & $71,88 \%$ & $28,12 \%$ & & $62,50 \%$ & $37,50 \%$ \\
Vitamina D & $84,38 \%$ & $15,62 \%$ & & $78 \%$ & $22 \%$ \\
Vitamina A & $84,38 \%$ & $15,62 \%$ & & $46,88 \%$ & $53,12 \%$ \\
\hline
\end{tabular}

Fonte: elaborada pelos autores (2017).

O processo educacional baseia-se em uma construção contínua, e não se encerra em apenas uma oficina, sendo necessário o desenvolvimento de ações educativas ao longo do processo, já que conhecimentos adequados sobre adoção de hábitos alimentares saudáveis deverão ser contemplados desde a infância (COSTA et al., 2016).

Para a avaliação de ações educativas faz-se necessário identificar e avaliar o número e a qualidade de atividades, visto que os aspectos didáticos influenciam no alcance dos objetivos propostos pelas intervenções no universo escolar (BERNARDON et al., 2009).

No terceiro encontro, foi realizada uma oficina culinária denominada "Vamos comer o peixe?". Para satisfazer as condições higiênico-sanitárias, cada criança recebeu um avental, touca e luvas e em seguida foram posicionadas ao redor de uma grande mesa, onde os utensílios estavam organizados e os ingredientes devidamente pré-preparados e proporcionados (Figura 05).

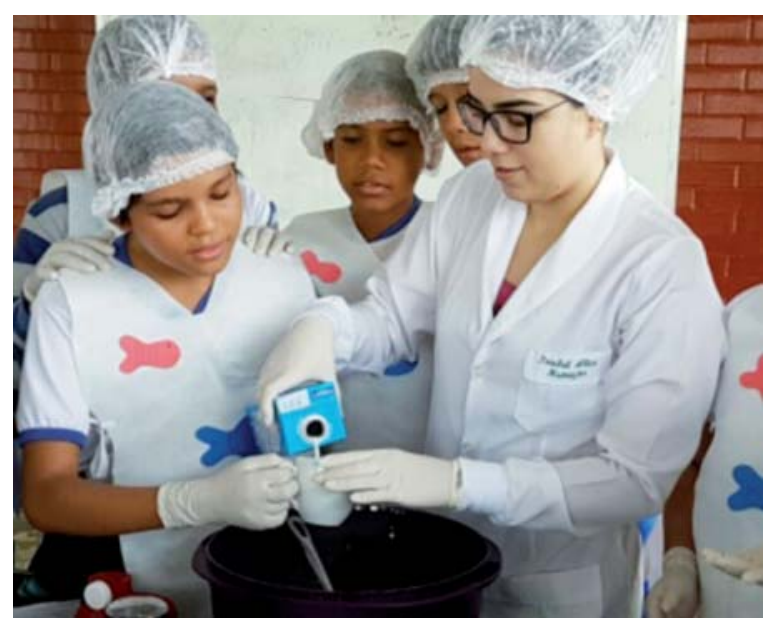

Figura 05- Alunos fazendo a torta

Fonte: arquivo próprio 
Esse momento oportunizou aos escolares uma participação ativa e em equipe, na preparação da receita, que tinha como o principal ingrediente o peixe.

As oficinas culinárias impactam positivamente na alimentação das crianças, pois, elas são incentivadas a conhecer e provar novos sabores (CRIBB, 2010). Além disso, tal atividade favorece a troca de experiência e permite o envolvimento dos alunos com as preparações, despertando o interesse do aluno para o consumo do produto final (COSTA et al., 2016)

Quando inseridas no ambiente escolar, as oficinas culinárias podem se tornar um método de ensino que proporciona uma relação direta entre alunos e alimentos, fomentando-se uma alimentação mais saudável, além de estender benefícios aos familiares e à comunidade envolvida, otimizando o trabalho com temas de educação alimentar e nutricional (MORGADO \& SANTOS, 2008; SILVA et al, 2015).

Costa et al. (2016) avaliaram a adoção de atividades lúdicas na promoção de alimentação no ambiente escolar, destacando a grande aceitação dos escolares em relação às oficinas culinárias. Os mesmos autores afirmaram que os escolares relataram fazer o preparo das receitas utilizadas nas oficinas em casa.

É importante ressaltar que as escolhas alimentares são experiências aprendidas. A familiaridade com o alimento é fator preponderante para sua aceitação e a partir daí aprende-se a gostar do que está disponível (FERNANDES, 2005).

\section{Conclusão}

Uma escola promotora de saúde estimula boas práticas de alimentação e incentiva a busca por escolhas alimentares conscientes e sustentáveis. Nesse sentido, considera-se fundamental discutir as abordagens teórico-metodológicas em educação alimentar para a construção de hábitos alimentares saudáveis.

As atividades de EAN aliadas as metodologias lúdicas de intervenção assumem papel decisivo no processo de mudanças das práticas alimentares dos escolares por despertar o interesse e curiosidade pelo alimento, e imprimir nas crianças desejos de consumi-lo, atitude essa que pode contribuir para boas escolhas que, quando aprendidas e adquiridas na infância, refletirão nas escolhas e estilo de vida na fase adulta.

A adoção de atividades lúdicas de intervenção oportunizaram a construção de conhecimento sobre a aquisição, composição e consumo de peixe, demostrando a aplicabilidade dos métodos pedagógicos utilizados nas oficinas, evidenciando que cada vez mais deve-se estimular a prática e aplicação de atividades direcionadas para o fortalecimento de conteúdos de nutrição.

\section{Fontes de financiamento}

O presente estudo não recebeu apoio financeiro.

\section{Referências}

BERNARDON, R.; SILVA, J.R.M.; CARDOSO, J.T.; MONTEIRO, R.A.; AMORIM, N.F.A.; SCHMITIZ, B.A.S.; RODRIGUES, M.L.C.F. Construção de metodologia de capacitação em alimentação e nutrição para educadores. Revista de Nutrição. v. 22, n.3, p. 389-398, 2009.

BRAGANÇA, F. A importância do peixe na nutrição. 2016. Disponível em: <http:// fernandobraganca.com.br/2016/06/03/a-importancia-do-peixe-na-nutricao/> Acesso: 15 de novembro de 2017.

BRASIL. Resolução No 26, de 17 de junho de 2013. Dispõe sobre o atendimento da alimentação escolar aos alunos da educação básica no âmbito do Programa Nacional de Alimentação Escolar - PNAE. Disponível em: <https://www.fnde.gov.br/fndelegis/action/UrlPublicasAction.php?acao=getAto Publico\&sgl_tipo $=$ RES\&num_ato $=00000026 \& s e q \_a t o=000 \& v l r \_a n o=2013 \& s g 1$ orgao $=F N D E /$ MEC> Acesso: 15 de novembro de 2017.

CASEMIRO, J. P.; FONSECA, A. B. C.; SECCO, F. V. M. Promover saúde na escola: reflexões a partir de uma revisão sobre saúde escolar na América Latina. Ciência \& Saúde Coletiva. v. 19. n. 3, p. 829-840, 2014. . Disponível em: http://www.scielosp.org/scielo.php?script=sci_arttext \&pid=S1413-81232014000300829 Acesso: 27/05/2017. DOI: 10.1590/1413-81232014193.00442013. 
CRIBB, S. L. S. P. Contribuições da educação ambiental e horta escolar na promoção de melhorias ao ensino, à saúde e ao ambiente. Revista Eletrônica do Mestrado Profissional em Ensino de Ciências da Saúde e do Ambiente. v.3, n. 1, p. 42-60, 2010.

COSTA, M.C.; SAMPAIO, E.V.; ZANIRATI, V.F.; LOPES, A.C.S.; SANTOS, L.C. Experiência lúdica de promoção de alimentação saudável no ambiente escolar: satisfação e aprendizado dos estudantes. $\mathrm{O}$ mundo da saúde. v. 40, n. 1, p.38-50, 2016.

FERNANDES, M.C.A. A Horta Escolar como eixo gerador de dinâmicas Comunitárias, Educação Ambiental e Alimentação Saudável e Sustentável. Brasília, 2005. Projeto PCT/BRA/3003 - FAO e FNDE/MEC.

FONSECA, L.M.M.; SCOCHI, C.G.S.; MELLO, D.F. Educação em saúde de puérperas em alojamento conjunto neonatal: aquisição de conhecimento mediado pelo uso de um jogo educativo. Revista Latinoamericana de Enfermagem. V. 10, n.02, p. 166-171, 2002.

FREIRE, P. Pedagogia da autonomia. São Paulo, editora Paz e Terra, 2007.

GARROCHO, L.C. Uma prática para a arte-educação. Revista Sala de Aula. v. 23, n. 3, p. 34- 41, 1990.

HEBERLE, K. Importância e utilização das atividades lúdicas na educação de jovens e adultos. Disponível em: <http://repositorio.roca.utfpr.edu.br/jspui/bitstream/1/1764/1/MD_PROEJA_2012 _IV_09.pdf> Acesso: 15 de novembro de 2017.

KATO, H.C. Peixe fresco: da compra ao preparo. Embrapa: Pesca e Aquicultura, Palmas, 2014.

KREBS-SMITH, S.M.; COOK, D.A.; SUBAR, A.F.; CLEVELAND, L.; FRIDAY, J.; KAHLE, L.L. Fruit and vegetable intakes of children and adolescents in the United States. Archives of Pediatrics \& Adolescent Medicine, v. 150, n.01, p. 81-86, 1996.

MORGADO, F.S.; SANTOS, M.A.A. A horta escolar na educação ambiental e alimentar: experiência do Projeto Horta Viva nas escolas municipais de Florianópolis. Extensio - Revista Eletrônica de Extensão. v.5, n.6, p. 1-10, 2008.

NETO, O.O.B. Programa Nacional de Alimentação Escolar - PNAE. Disponível em: <http:// litoralsustentavel.org.br/wp-content/uploads / 2015/10/Apresenta \% C3\% A7\% C3\% A3o Pescado_Olavo_COSAN_Pescado1.pdf> Acesso: 15 de novembro de 2017.

ORDÓÑEZ, J. A. Tecnologia de alimentos: alimentos de origem animal. Porto Alegre: Artmed, 2005. v. 2, cap. 11.

ROSSI, A.; MOREIRA, E.A.M.; RAUREN, M.S. Determinantes do comportamento alimentar: uma revisão com enfoque na família. Revista de Nutrição, v. 21, n. 06, p. 739-748, 2008.

SARTORI, A.G.O.; AMANCIO, R.D. Pescado: importância nutricional e consumo no Brasil. Segurança alimentar e nutricional, v. 19, n. 2, p. 83-93, 2012.

SILVA, C.F.M.; PINTO, M.S.; PIRES, C.R.F.; KATO, H.C Relato de experiência de educação nutricional para o incentivo do consumo do pescado entre escolares do ensino fundamental em Palmas, Tocantins. Em Extensão. v. 16, n.1, p. 140-149, 2017. Disponível em: <http://www.seer.ufu.br/index.php/ revextensao/article/viewFile/37874/pdf> Acesso: 15 de novembro de 2017.

SILVA, A.C.D.; SOUSA, A.A.; NASCIMENTO, C.R. Horta na escola: sustentabilidade e hábitos saudáveis no município de Cantá-RR. Atas de Saúde Ambiental. v.3, n.3, p.80-89, 2015.

TURANO, W.; ALMEIDA, C. C. C. A. Educação Nutricional. In: GOUVEIA, E. L. C. Nutrição, Saúde e Comunidade. 2. ed. Rio de Janeiro: Revinter, 1999. p. 57-77.

TURANO, W. A Didática na Educação Nutricional. In: GOUVEIA, E. Nutrição Saúde e Comunidade. São Paulo: Revinter, 1990. 246 p.

VIVAS, E.; SEQUEDA, M.G. Um juego como estrategia educativa para el control de Aedes aegypti en escolares venezolanos. Revista Panamericana de Salud Pública, v. 14, n. 6, p. 394-401, 2003.

WINNICOTT, D. W. O Brincar e a realidade. Rio de Janeiro: Imago, 1975.

Recebido para publicação em 17/2/2018 e aprovado em 13/6/2018. 